\section{Gente Sana en Comunidades Saludables: la visión de Salud para Todos en los Estados Unidos de América}

\author{
Cristina Puentes-Markides, ${ }^{1}$ \\ Deborah Maiese ${ }^{2}$ \\ y Myriam Granthon ${ }^{3}$
}

\footnotetext{
1 Oficina de Análisis y Planificación Estratégica, Organización Panamericana de la Salud. Dirección postal: 525 Twenty-third Street, N.W., Washington, DC 20037, EE.UU.

2 Office of Disease Prevention and Health Promotion, U.S. Department of Health and Human Services, Washington, DC, EE.UU.

3 Healthy People Consortium, Office of Disease Prevention and Health Promotion, U.S. Department of Health and Human Services, Washington, DC, EE.UU.

4 Dirección electrónica: http//www.health.gov/healthypeople
}

Gente Sana 2000 (Healthy People 2000), política actual de promoción de la salud y prevención de enfermedades de los Estados Unidos de América, es un conjunto de objetivos nacionales basados en un plan de actividades, una visión y una serie de metas audaces para lograr adelantos en el campo de la salud. Esta política se extenderá durante el período 2000-2010 bajo la designación de Gente Sana 2010. ${ }^{4}$

Durante los últimos 20 años, los Estados Unidos han elaborado metas nacionales cuantificables para promover la salud y prevenir enfermedades. La experiencia ha revestido especial interés por su naturaleza participatoria, reflejada en el desarrollo de políticas de salud, la creación de alianzas entre una amplia variedad de sectores, el apoyo político bipartidario, la utilización de pruebas científicas y el gran fortalecimiento de los procesos de recolección de datos. Estos logros han sido posibles en una nación de enorme extensión territorial, con una población de más de 250 millones de habitantes de una enorme heterogeneidad étnica y racial y grandes disparidades en materia de salud. La amplia aceptación y adopción de los objetivos de Gente Sana por prácticamente todos los estados del país reafirma su validez en contextos socioeconómicos y culturales diversos.

La primera generación de objetivos se plasma en el documento Healthy People: the Surgeon General's Report on health promotion and disease prevention (1), publicado en 1979 y seguido un año más tarde de un volumen adicional, Promoting health/preventing disease: objectives for the nation (2), donde aparecían metas específicas por grupos de edad. Cinco de los objetivos actuales guardan relación con el ciclo de vida y se orientan a reducir la mortalidad prematura; el sexto busca preservar la independencia del adulto mayor. Para el 2010 se propone un enfoque denominado Gente Sana en Comunidades Saludables con dos objetivos generales para la nación: aumentar los años de vida saludable y de calidad y eliminar disparidades en materia de salud (3). Se han establecido 28 áreas prioritarias y más de 400 metas que dan origen a iniciativas comunitarias, motivan y cohesionan, y suscitan una estrecha comunicación entre el público, las autoridades de salud y los líderes comunitarios.

Los complejos patrones demográficos y epidemiológicos actuales y esperados exigen acciones integradas para toda la nación. El envejecimiento de la población y su profunda diversidad étnica y ra- 
cial, sumados a las posibilidades de vivir más tiempo con enfermedades crónicas, imponen la necesidad de invertir en actividades preventivas y de promoción de la salud. Se busca modificar la organización y el financiamiento de los servicios de salud, así como los sistemas de comunicación, de tal manera que a los consumidores les resulte más fácil obtener información para poder tomar mejores decisiones y tener acceso a servicios de calidad. Estas medidas son parte de una serie de estrategias diseñadas e implementadas mediante la acción conjunta de asociaciones, comunidades, familias e individuos. Para mejorar ostensiblemente la salud de la población, Gente Sana debe ser un paradigma de cambios no solo en cuanto a resultados en salud, sino también en lo referente a la asignación y utilización de recursos humanos, financieros y tecnológicos para actividades de promoción de la salud y prevención de enfermedades.

\section{La participación}

Con cada generación de Gente Sana (1979, 1980, 1987-1990, 1996-2000) se han incorporado actores y sectores -agricultura, educación, medio ambiente, transporte, etc. - interesados en mejorar la salud nacional de forma mancomunada. El logro de resultados a largo plazo requiere la participación activa de los ciudadanos, los proveedores de atención, las escuelas, los empleadores, la industria y las comunidades, y no solo el Gobierno.

El uso extenso de la Internet ha permitido la comunicación directa con el público en general. De hecho, en 1997 y 1998 este medio se empleó para recibir comentarios sobre el marco propuesto, los objetivos y las metas de Gente Sana 2010. Además de los testimonios públicos, cinco reuniones regionales (en Filadelfia, Chicago, Nueva Orleans, Seattle y San Francisco) ofrecieron a las comunidades la oportunidad de evaluar la utilidad del marco de las políticas y de expresar su opinión. Las consultas al público se incrementaron con la creación del Consorcio Gente Sana (Healthy People Consortium), resultado de un contrato entre el Servicio de Salud Pública de los Estados Unidos y el Instituto de Medicina de la Academia Nacional de Ciencias.

En 1987, 157 organizaciones nacionales y entidades de salud pública estatales y territoriales se habían sumado a la iniciativa y más de 10000 personas participaron en audiencias públicas u opinaron sobre el contenido de los objetivos y las metas de Gente Sana 2000. En la actualidad, el Consorcio cuenta con más de 350 asociados nacionales, lo que representa un total de 59 millones de personas. El Consorcio abarca, además, todos los departamentos de salud pública estatales, las agencias de salud mental, las autoridades estatales contra el abuso de sustancias y las agencias de asuntos ambientales. Esta entidad, de la cual es miembro la OPS, se reúne anualmente y produce boletines trimestrales con información sobre las actividades de los sectores estatal y privado en relación con los objetivos establecidos. El Healthy People Business Advisory Council [Consejo Asesor sobre Asuntos Relacionados con la Iniciativa de Gente Sana], entidad ajena al Consorcio, establece el nexo con el sector privado y le confiere a Gente Sana su valor como instrumento para la promoción de la salud en ámbitos laborales y comunitarios. Un miembro del Consorcio, la Fundación Robert Wood Johnson, incorpora activamente a empresarios norteamericanos al proceso de Gente Sana mediante su programa denominado Partnership for Prevention.

\section{Sustentabilidad política}

Gente Sana ha sido apoyada por administraciones republicanas y demócratas que le han dado visibilidad y mayor aplicación bajo los gobiernos de cuatro presidentes: Carter, Reagan, Bush y Clinton. En el ámbito estatal, los gobernadores respaldan sus objetivos y metas, y casi todos los estados de la unión, Guam y el Distrito de Columbia han publicado su propia versión de las metas para el año 2000. El trabajo incluso ha comenzado en muchos estados para el 2010.

La definición del marco general y de las metas de Gente Sana cuenta con la creciente participación de funcionarios del gobierno, expertos del gremio académico, líderes de grupos con intereses especiales, el sector privado y el sector público. También se han formado algunas alianzas entre grupos con características étnicas o raciales en común, problemas de salud especiales o una misma afiliación socioeconómica, hecho que fortalece la sustentabilidad política y la renovación de las actividades programadas. La participación activa de los sectores público y privado demuestra que el enfoque nacional es valioso, permite lograr un consenso en torno a metas específicas y repercute en las acciones de salud en el nivel local.

\section{Mecanismos de coordinación}

La propia elaboración de Gente Sana 2010 ha sido un proceso vinculador en el ámbito gubernamental en virtud de varios mecanismos. Uno de ellos es el Secretary's Council for National Health Promotion and Disease Prevention Objectives for 2010, encabezado por la Secretaría de Salud con la participación de los subsecretarios de salud de cuatro 
administraciones anteriores y los actuales jefes de agencias del Departamento de Salud y Servicios Humanos de los Estados Unidos (USDHHS). El Comité Directivo de Gente Sana (Healthy People Steering Committee), que celebra reuniones cuatrimestrales, es presidido por el Director de la Oficina de Prevención de Enfermedades y Promoción de Salud y está integrado por los coordinadores de los grupos de trabajo para cada una de las 15 áreas de Gente Sana 2000 que representan las agencias principales. Las reuniones de evaluación periódica de cada una de las áreas, presididas por el Subsecretario de Salud o el Cirujano General, constituyen otro instrumento articulador, puesto que refuerzan la responsabilidad de cada participante en materia de vigilancia, evaluación de la eficacia de los programas y revisión de las estrategias para mejorar los resultados.

\section{Vigorización de los procesos de desarrollo de políticas de salud}

Gente Sana no es una receta; constituye, más bien, un menú nacional del cual puede elegir cada estado, condado o localidad a la luz de sus problemas específicos, factibilidad financiera o viabilidad política. La obtención de datos de calidad es uno de los mayores desafíos, ya que para establecer valores de base o referencia que permitan vigilar los adelantos se necesita información sobre zonas geográficas pequeñas y grupos de población particulares. El establecimiento de metas mensurables y con valores de referencia básicos ha dado ímpetu a un proceso importante para la elaboración de datos. Puede afirmarse, por consiguiente, que Gente Sana formenta una mejor recolección y utilización de datos en materia de salud. Las brechas existentes en el campo de la información han dado lugar a iniciativas para llamar la atención al problema e impulsar aquellas áreas que necesitaban mayor desarrollo. Los suplementos de encuestas establecidas, como la Encuesta Nacional de Entrevistas de Salud (National Health Interview Survey) y el Sistema para la Vigilancia de Comportamientos Riesgosos (Surveillance System for Risky Behaviors), han aportado información complementaria sobre los programas y las prácticas en el ámbito de la salud escolar, las actividades de promoción de la salud en el lugar de trabajo, y las actividades de prevención, asesoramiento de pacientes y evaluación de la salud por parte de los médicos generales.

\section{Magnitud global y perspectivas a largo plazo}

En el marco de Gente Sana se reconoce que fomentar la salud y eliminar las enfermedades y los trastornos que la afectan incumben cada vez más a la comunidad mundial, debido a la interdependencia de diversos factores transnacionales. Las enfermedades transmisibles, la contaminación de los alimentos, el terrorismo biológico y el uso de sustancias tóxicas se ven facilitados por el movimiento transfronterizo de personas, mientras que la expansión de las redes de comunicación fomenta la diseminación de comportamientos sociales o sexuales de riesgo. La formación de profesionales de la salud, la armonización de normas reguladoras, los derechos de propiedad y el establecimiento de sistemas de vigilancia han dejado de ser asuntos puramente nacionales.

La inclusión de escenarios futuros donde se examinan las perspectivas de lograr los objetivos y las metas de Gente Sana 2010 ofrece a los gobiernos una oportunidad enriquecedora para mejorar su capacidad predictiva. Una perspectiva futurista y estratégica permite mayor flexibilidad y adaptabilidad frente a las transformaciones de la ciencia, la tecnología, la medicina y los aspectos sociales que las acompañan.

\section{Actividades concomitantes}

La Iniciativa de la Raza (Race Initiative), lanzada en 1997 por el Presidente Clinton, se refleja en el objetivo del USDHHS de eliminar las desigualdades en materia de salud. Existen abundantes pruebas de que la raza y el origen étnico se correlacionan con disparidades persistentes en el ámbito de la salud y de que estas disparidades se están incrementando en determinados grupos de población. Esta iniciativa en pro de la salud se concentrará en seis áreas donde hay serias discrepancias en el acceso a la atención de salud y en los resultados de la misma: mortalidad infantil, diagnóstico y tratamiento del cáncer, enfermedades cardiovasculares, diabetes, VIH y sida e inmunización de niños y adultos.

Un trabajo comisionado por el USDHHS al Instituto de Medicina (Academia Nacional de Ciencias) sobre los indicadores centinela para Gente Sana 2010, iniciado en 1997, constituye otra medida de importancia. Como resultado de ella se produjeron grupos de indicadores de salud relevantes que podrían llevar al público a sentirse más comprometido con el logro de comportamientos saludables.

Gente Sana 2010 se ha articulado con el movimiento de Comunidades Saludables (Healthy Communities). La Oficina de Prevención de Enfermedades y Promoción de la Salud ha publicado una guía, titulada Resource Guide for Healthy Communities/ Healthy Cities (4), para apoyar el desarrollo de objetivos de base comunitaria en escuelas, lugares de trabajo y servicios de salud.

Gente Sana se ha extendido más allá de las fronteras estadounidenses. La experiencia se pre- 
sentó en febrero de 1998 durante un Simposio sobre Estrategias Nacionales de Salud para Todos (Symposium on National Strategies for Health for All) que se celebró en Washington, D.C. (5) con la participación de representantes de 17 países americanos. En dicha ocasión varios países manifestaron su interés en un trabajo de colaboración conjunta con los Estados Unidos encaminado a establecer políticas para la promoción de la salud y la prevención de enfermedades.

\section{Conclusiones}

Lograr la salud para todos es la visión global de Gente Sana 2010, iniciativa con la cual los Estados Unidos se sumarán al llamado de la OMS y de la OPS de renovar la meta de Salud para Todos. Como expresión nacional de esta visión, Gente Sana encierra varios compromisos: lograr la equidad mediante la eliminación de disparidades en el campo de la salud; conseguir la solidaridad mediante un enfoque centrado en la comunidad; y lograr la sustentabilidad mediante un esfuerzo conjunto del gobierno, la sociedad, la comunidad, las familias y los individuos. Gente Sana 2010 será lanzada públicamente durante una conferencia patrocinada por el Consorcio Gente Sana y Partnerships for Networked Consumer Health Information, que se celebrará en Washington, DC, del 24 al 28 de enero de $2000 .{ }^{4} \mathrm{Du}-$ rante este evento se darán a conocer los objetivos de Gente Sana 2010; se promoverán alianzas para lograr la visión de Gente Sana 2010, Gente Sana en Comunidades Sanas, y Salud para Todos; se subrayará la utilización efectiva de la tecnología para promover la salud en lo individual y colectivo; y se vigorizará a grupos con intereses específicos para que dirijan las acciones en pro de la salud en el siglo XXI. Las comunidades de todo el país plantearon los objetivos que han trazado para la semana dedicada a la salud durante el mes de abril de 2000, con lo cual se consolidó un marco coherente para el mejoramiento de la salud en el ámbito nacional.

Podría afirmarse que Gente Sana 2010 es una iniciativa "del pueblo, por el pueblo y para el pueblo" y un gran desafío para los Estados Unidos. La vigilancia y medición de disparidades y el establecimiento de medidas de salud ambiental y social exi- gen la elaboración de objetivos tangibles, así como la responsabilidad y el compromiso de las comunidades y gobiernos en el logro de salud. La audacia de algunas metas servirá para consolidar el compromiso, estimular la imaginación, redoblar los esfuerzos e incrementar los recursos, reclamando por estos medios inversiones reales en ganancias en salud. El plan de trabajo para la próxima década es parte de una nueva visión de la salud: su consecución por todos y cada uno de los habitantes del planeta.

\section{SYNOPSIS}

\section{Healthy People in Healthy Communities: the vision of Health for All in the United States of America for 2000-2010}

For the first decade of the new millennium, "Healthy People 2010 " will be the official designation of the health promotion and disease prevention policy of the United States of America. The policy's work plan carries on the vision of Health for All that is promoted by the World Health Organization and the Pan American Health Organization and whose attainment will be the responsibility of each and every person on earth.

Healthy People in Healthy Communities, as the approach to Healthy People 2010 will be called, will have two general objectives for the United States as a whole. One is to increase the years of healthy life, incorporating the concept of quality of life into that of healthy life expectancy. The second objective is to eliminate health disparities. Twenty-eight priority areas and more than 400 goals will be consolidated through community initiatives and close communication among the public, health authorities, and community leaders.

The experience of the United States is particularly interesting because it is participatory, as shown by the creation and consolidation of alliances between numerous sectors, bipartisan political support, use of scientific tests to support decisions, and efforts to strengthen data collection processes. The broad acceptance and adoption of the objectives of Healthy People by practically all fifty states reaffirms the initiative's validity in various socioeconomic and cultural contexts. Healthy People 2010 will be launched publicly during a conference that will be held in Washington, D.C., from 24 to 28 January 2000, with support from the Healthy People Consortium and the Partnerships for Networked Consumer Health Information.

\section{REFERENCIAS}

1. Public Health Service. Healthy People: The Surgeon General's Report on Health Promotion and Disease Prevention. Washington, DC, 1979. U.S. Department of Health and Human Services, 1979.

2. U.S. Department of Health and Human Services, Office of Disease Prevention and
Health Promotion. Promoting health/ preventing disease: objectives for the nation. Washington, DC: USDHHS, ODPHP; 1980.

3. U.S. Department of Health and Human Services, Office of Disease Prevention and Health Promotion. Developing Objectives for Healthy People 2010. Washington, DC: USDHHS, ODPHP; September 1997.

4. U.S. Department of Health and Human Services, Office of Disease Prevention and Health Promotion. Resource guide for healthy communities/healthy cities. Washington, DC: USDHHS, ODPHP; 1988. 